\title{
Assessing the effect of interventions for axial spondyloarthritis according to the endorsed ASAS/OMERACT core outcome set: a meta-research study of trials included in Cochrane reviews
}

Rikke A. Andreasen 1,201, Lars E. Kristensen ${ }^{2}$, Xenofon Baraliakos ${ }^{3}$, Vibeke Strand ${ }^{4}$, Philip J. Mease ${ }^{5}$, Maarten de Wit ${ }^{6}$, Torkell Ellingsen ${ }^{7}$, Inger Marie J. Hansen ${ }^{1}$, Jamie Kirkham ${ }^{8}$, George A. Wells ${ }^{9}$, Peter Tugwell ${ }^{10}$, Lara Maxwell ${ }^{10}$, Maarten Boers ${ }^{11}$, Kenneth Egstrup ${ }^{12}$ and Robin Christensen ${ }^{2,7^{*}}$ (D)

\begin{abstract}
The Assessment of SpondyloArthritis international Society (ASAS) has defined core sets for (i) symptom-modifying anti-rheumatic drugs (SM-ARD), (ii) clinical record keeping, and (iii) disease-controlling anti-rheumatic therapy (DCART). These include the following domains for all three core sets: "physical function," "pain," "spinal mobility," "spinal stiffness," and "patient's global assessment" (PGA). The core set for clinical record keeping further includes the domains "peripheral joints/entheses" and "acute phase reactants," and the core set for DC-ART further includes the domains "fatigue" and "spine radiographs/hip radiographs." The Outcome Measures in Rheumatology (OMERACT) endorsed the core sets in 1998.

Using empirical evidence from axSpA trials, we investigated the efficacy (i.e., net benefit) according to the ASAS/ OMERACT core outcome set for axSpA across all interventions tested in trials included in subsequent Cochrane reviews. For all continuous scales, we combined data using the standardized mean difference (SMD) to metaanalyze outcomes involving the same domains. Also, through meta-regression analysis, we examined the effect of the separate SMD measures (independent variables) on the primary endpoint (log $[O R]$, dependent variable) across all trials.

Based on 11 eligible Cochrane reviews, from these, 85 articles were screened; we included 43 trials with 63 randomized comparisons. Mean (SD) number of ASAS/OMERACT core outcome domains measured for SM-ARD/ physical therapy trials was 4.2 (1.7). Six trials assessed all proposed domains. Mean (SD) for number of core outcome domains for DC-ART trials was 5.8 (1.7). No trials assessed all nine domains. Eight trials (16\%) were judged to have (Continued on next page)
\end{abstract}

\footnotetext{
* Correspondence: Robin.Christensen@regionh.dk

${ }^{2}$ Musculoskeletal Statistics Unit, the Parker Institute, Bispebjerg and Frederiksberg Hospital, University Hospital, Copenhagen F, Denmark ${ }^{7}$ Research Unit of Rheumatology, Department of Clinical Research, University of Southern Denmark, Odense University Hospital, Odense, Denmark Full list of author information is available at the end of the article
}

C C The Author(s). 2020 Open Access This article is licensed under a Creative Commons Attribution 4.0 International License, which permits use, sharing, adaptation, distribution and reproduction in any medium or format, as long as you give appropriate credit to the original author(s) and the source, provide a link to the Creative Commons licence, and indicate if changes were made. The images or other third party material in this article are included in the article's Creative Commons licence, unless indicated otherwise in a credit line to the material. If material is not included in the article's Creative Commons licence and your intended use is not permitted by statutory regulation or exceeds the permitted use, you will need to obtain permission directly from the copyright holder. To view a copy of this licence, visit http://creativecommons.org/licenses/by/4.0/. The Creative Commons Public Domain Dedication waiver (http://creativecommons.org/publicdomain/zero/1.0/) applies to the data made available in this article, unless otherwise stated in a credit line to the data. 


\begin{abstract}
(Continued from previous page)
inadequate (i.e., high risk of) selective outcome reporting bias. The most responsible core domains for achieving success in meeting the primary objective per trial were pain, OR (95\% Cl) $5.19(2.28,11.77)$, and PGA, OR $(95 \% \mathrm{Cl})$ $1.87(1.14,3.07)$. In conclusion, selective outcome reporting (and "missing data") should be reduced by encouraging the use of the endorsed ASAS/OMERACT outcome domains in clinical trials. Overall outcome reporting was good for SM-ARD/physical therapy trials and poor for DC-ART trials. Our findings suggest that both PGA and pain provide a valuable holistic construct for the assessment of improvement beyond more objective measures of spinal inflammation.
\end{abstract}

Keywords: Axial spondyloarthritis, Ankylosing spondylitis, Core outcome set, Meta-analysis

\section{Introduction}

Since 1992, the Outcome Measures in Rheumatology (OMERACT) consensus initiative has successfully developed core-or minimum-sets for many rheumatologic conditions [1]. A "core outcome set" (COS) represents which outcome domains (i.e., constructs or concepts [what to measure]) and outcome measurements (i.e., how to measure]) to apply in RCTs [2].

ASAS has aimed to bring evidence-based unity to the multitude of assessments in the field of axial spondyloarthritis (axSpA). Currently, ASAS's scope includes the entire spectrum of SpA [3]. axSpA comprises two subcategories based on the presence of structural changes in the sacroiliac joints: radiographic ( $r-$ ) axSpA, implying the fulfillment of the modified New York criteria, and non-radiographic (nr) axSpA.

ASAS has selected a set of core outcome domains to include among a set of standardized measures in clinical trials, which is defined by the following scenarios: (i) symptom-modifying anti-rheumatic drugs (SM-ARD)/physiotherapy, (ii) clinical record keeping for studies, and (iii) disease-controlling anti-rheumatic therapy (DC-ART) (Fig. 1). The selected domains to include as standardized outcomes in RCTs for all three scenarios include the following: "physical function," "pain," "spinal mobility," "spinal stiffness," and "patient's global assessment" (PGA). The core set for clinical record keeping further includes the domains "peripheral joints/entheses" and "acute phase reactants," and the core set for DC-ART further includes the domains "fatigue" and "spine and hip radiographs" [4]. ASAS core outcome domain sets were endorsed by OMERACT in 1998 [5].

Although composite outcomes seem an attractive method to increase statistical power (e.g., BASDAI 50 response), they can mask the effect of (or absence of) the individual domains of treatment. This study therefore sets out to assess the effect of interventions for axSpA according to each core domain in the existing COS, as well as its association with the primary statistical outcome in the individual trials.

\section{Main text}

Materials and methods

We conducted a meta-epidemiological study by evaluating axSpA trials included in Cochrane reviews (i.e., Cochrane Musculoskeletal Review Group). Study selection, assessment of eligibility criteria, data extraction, and statistical analyses were performed based on a pre-specified protocol. In accordance with current methodology, the protocol is available (Supplement A) and registered on PROSPERO (CRD42018091257). The study conforms to the Preferred Reporting Items for Systematic Reviews and Meta-Analyses (PRISMA) guidelines for reporting systematic reviews and metaanalyses $[6,7]$.

\section{Literature search}

A systematic search was done on May 1, 2018, to identify all Cochrane reviews that reported interventions for the management of axSpA. Two reviewers (RAA and RC) searched directly in the Cochrane Database of Systematic Reviews, where eligible trials were identified from published Cochrane reviews (i.e., meta-analyses) after a thorough search, using the following terms: (ankylosing spondylitis OR bechterew disease OR ankylosing spondylarthritides OR axial spondyloarthritis OR axial spondyloarthritides). The most recent version of the Cochrane review was used. Unlike what was pre-specified in PROSPERO, for feasibility, we used the Cochrane Database of Systematic Reviews directly rather than PubMed, since this meta-research study's eligibility criteria state that only trials included in Cochrane reviews would be considered for eligibility.

\section{Eligibility criteria}

Cochrane reviews that incorporated RCTs in patients with axSpA were included in our study. Only reviews with superiority trials were considered eligible. All reports for each RCT included in eligible reviews were obtained for evaluation. Non-RCTs and trials without full publications were excluded. 


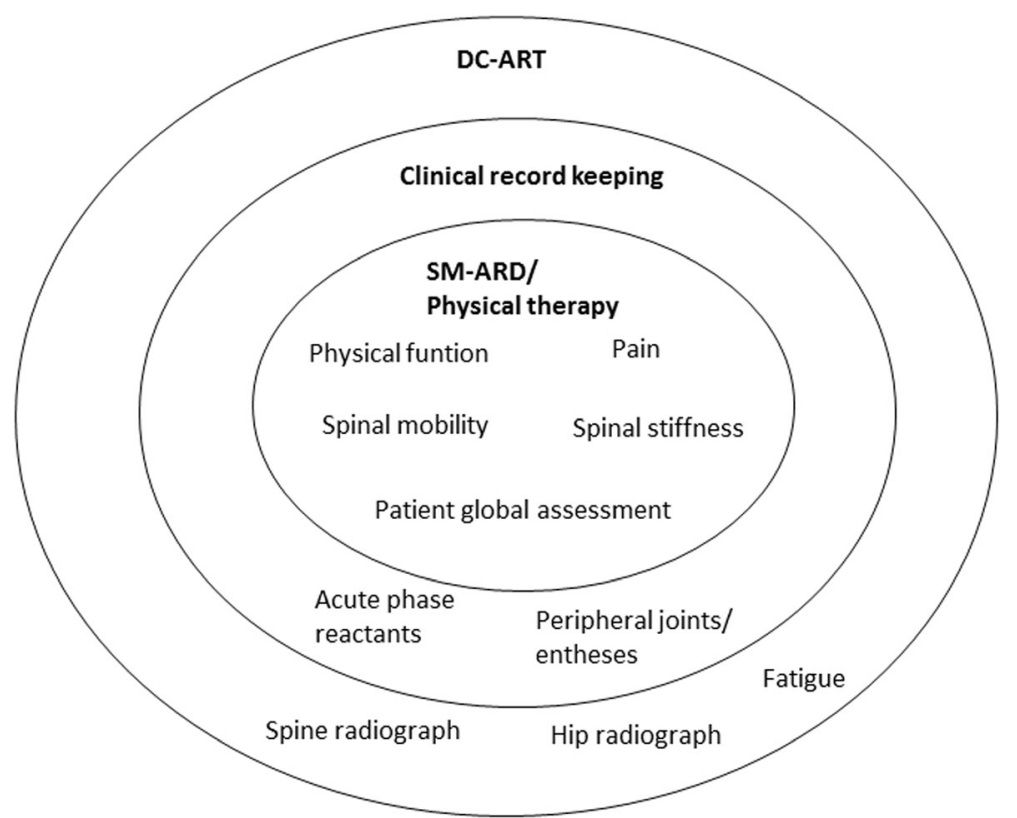

Fig. 1 ASAS/OMERACT core domains for axSpA. Inner circle, core domains for SM-ARD/physical therapy; two inner circles, core domains for clinical record keeping; all three circles, core domains for DC-ART. SM-ARD, symptom-modifying anti-rheumatic drug; DC-ART, disease-Controlling anti-rheumatic treatment

\section{Risk of bias in individual studies (internal validity)}

The risk of bias (RoB) within each study was assessed using the domains of the RoB tool, as recommended by the Cochrane Collaboration [8]. The bias domains included selection bias (methods for sequence generation and allocation concealment), performance bias (blinding of participants and personnel), attrition bias (incomplete outcome data), and reporting bias (selective outcome reporting). Each domain was rated as adequate, inadequate, or unclear risk of bias [9]. RAA completed all the RoB assessments and applied the RoB that was included and reported in the original Cochrane reviews as a proxy for a second reviewer assessment.

\section{Data extraction strategy}

At trial level, the terms of extraction comprised information about the first author, publication year, study duration, type of intervention, and total number of patients randomized.

The domains that were collected included the following: (i) physical function, (ii) pain, (iii) spinal mobility, (iv) spinal stiffness, (v) fatigue, (vi) patient's global assessment, (vii) peripheral joints/entheses, (viii) acute phase reactants, and (ix) spine and hip radiographs. Furthermore, at the individual trial level, we extracted data on how many participants achieved the stated primary outcome.

If data on more than one instrument was provided for any domain, we extracted data on the scale highest on the list proposed by ASAS/OMERACT [3, 4] (Supplement B).
Trials with multiple intervention arms were treated as individual trials, referred to as "randomized comparisons" (i.e., three-arm trials with two active interventions generated two randomized comparisons with placebo). However, the number of patients in the placebo groups was divided by the number of active treatment arms, thus adjusting the standard errors in order to avoid double counting of patients [10].

\section{Statistical analysis}

Treatment effect sizes for all domains were expressed as standardized mean differences (SMDs) [11]. Standard pairwise meta-analyses for the nine domains' SMDs with the corresponding 95\% confidence interval (CI) were performed with Review Manager (version 5.3). Negative SMD values indicated a beneficial effect of the experimental intervention (e.g., pain reduction) compared with control comparator; for ease of interpretation, we used the following "rule of thumb": SMDs of more than 0.2 represents a small effect, 0.5 a moderate effect, and 0.8 a large effect [12].

We used standard random-effect meta-analysis as the default option, whereas the fixed-effect analysis was applied for the purpose of sensitivity [13]. We used the chi ${ }^{2}$ test (Cochrane's $Q$ test) to assess heterogeneity and the $I^{2}$ statistic to assess inconsistency $[8,13]$. Anticipating substantial heterogeneity, a pre-specified number of stratified and meta-regression analyses were planned. We conducted the following stratified analyses to examine the influence of different subgroups-Pharmacological vs. Non- 
pharmacological treatment, and Biologic vs. Other treatment-on the effect of the interventions for all the core outcomes. Only covariates that reduced the variation (decrease in the $\tau^{2}$ estimated as tau squared $\left.\left[T^{2}\right]\right)$ seen in the estimates across strata were considered potentially relevant. In trials where the primary outcome was a composite outcome, meta-regression was performed to investigate which of the nine core domains (via the available SMDs) were best associated with the primary composite endpoint of the individual trials $\left(\log \left[O R_{\mathrm{i}}\right]\right)$. Meta-regression was performed in a stepwise manner with the following three steps:

1. Each of the core domains was analyzed as the only independent variable in a univariate metaregression analysis concerning the effect of the domains on the odds ratio (OR) for achieving the primary endpoint. Arbitrarily, it was decided that variables with a $P$ value $>0.05$ were excluded as potential explanatory variables in step 3 . The analyses were based on all trials reporting the primary endpoint.

2. The univariate meta-regression analysis mentioned above was repeated, but only trials reporting all the domains affecting the $\log \left[O R_{\mathrm{i}}\right](P<0.05)$ were included in the analysis.
3. The explanatory core domains from step $1(P<$ $0.05)$ were analyzed as the independent variables in a multivariate meta-regression analysis.

These meta-regression analyses enabled us to explore which core domains were best reflected in the composite endpoint of axSpA and what is lost when we neglect core domains by using only one composite outcome endpoint.

\section{Patient perspective}

As part of the author team, MdW-an experienced patient research partner (PRP)-was consulted to review and elaborate on the protocol and confirmed the importance of the study from the patient's perspective. MdW was involved throughout the research process as a scientific collaborator and voluntarily participated in the process of designing and preparing the study protocol and in interpreting results. Where feasible, we followed the EULAR recommendations for PRPs [14].

\section{Results}

\section{Study selection}

The search was carried out directly in the Cochrane Library on May 01, 2018. As illustrated in Fig. 2, the

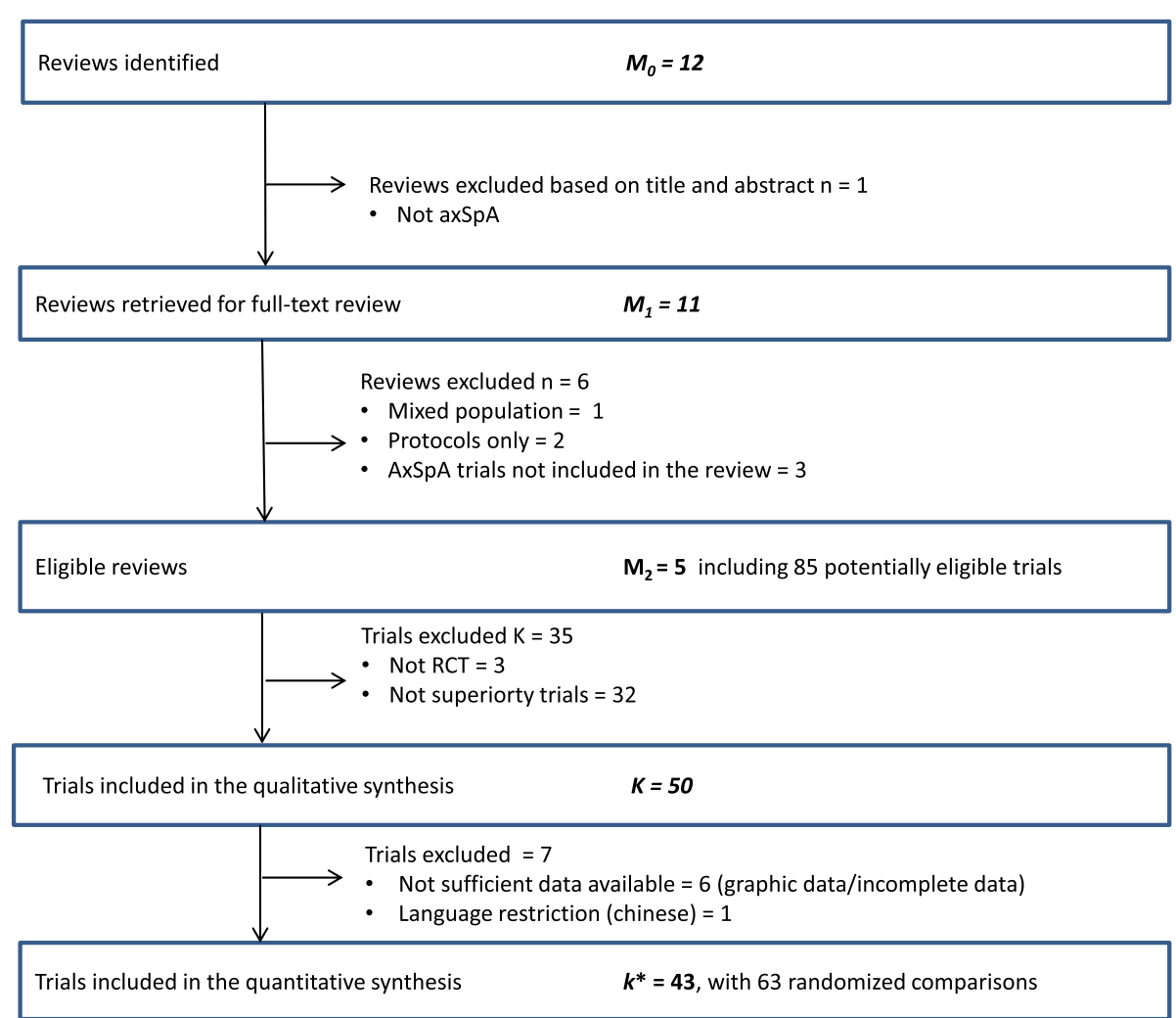

Fig. 2 Flow chart. $M_{0}$, identified Cochrane reviews; $M_{1}$, possible eligible reviews; $M_{2}$, included reviews; $K$, trials from included Cochrane reviews, $k^{*}$, trials included in the evidence synthesis 
inclusion criteria identified twelve Cochrane reviews; one review was excluded based on the title and abstract [15]. Eleven reviews were thus retrieved for full-text examination [16-26]. After full-text examination, we excluded another six reviews (three reviews did not include axSpA trials [21-23]; two reviews were protocols only $[25,26]$; one review did not report results for axSpA separately [24]). A total of five Cochrane reviews [16-20] with 85 possible trials were identified for inclusion. We excluded 35 studies -3 were not RCTs and 32 were not superiority trials-thus, 50 trials were found eligible for the qualitative synthesis (for reference list of included studies, see Supplement B). Of these 50 trials, 7 were not included in the quantitative synthesis: 6 trials reported most of the data as graphs, and data were not extracted [27-32], and one trial was excluded due to language restriction (Chinese [33]). The 43 included RCTs comprised a total of 63 comparisons. The interventions were categorized into three treatment groups: non-pharmacological (NP) modalities, pharmacological (P) modalities, and biological (B) modalities.

\section{Study characteristics}

The characteristics of the eligible trials are summarized in Table 1.

Twenty-two trials (42\%) used an adequate concealment of allocation and sequence generation (selection). Twenty-seven trials (54\%) were judged to have adequate blinding of participants and caregivers (performance), and 34 trials (68\%) adequately addressed incomplete outcome data (attrition). Eight trials [29, 34-40] (16\%) were unable to provide the data of all the pre-specified outcomes, and we judged them at high risk of selective outcome reporting bias.

\section{Characteristics of the core outcome measurement set}

The outcome matrix (Table 2) shows which core domains were measured for each trial and by which measurement instrument, differentiating between those which were fully and partially reported. Overall outcome reporting was good for SM-ARD/physical therapy trials; mean (SD) number of ASAS/OMERACT core outcome domains measured for SM-ARD/ physical therapy trials was 4.2 (1.7), and six trials assessed all five proposed domains. Mean (SD) number of ASAS/OMERACT core outcome domains measured for DC-ART trials was 5.3 (1.8). No DC-ART trial assessed all nine domains. The most commonly measured domain was spinal mobility (88\%) which was assessed followed by pain (86\%). Most studies also included measures of physical function (78\%), spinal stiffness (76\%), acute phase reactants (70\%), and patient's global assessment (62\%). The instruments used to measure the domains varied widely across trials. For the domain fatigue, only seven trials (14\%) had reported this measure separately. Spine radiographs were also poorly represented $(2 \%)$. None of the trials reported hip radiographs.

\section{Physical function}

All meta-analyses are shown in Supplement B.

A total of 33 RCTs (43 comparisons, 4819 participants) were included in the meta-analysis. As presented in Table 3, the overall analysis of change in physical function (PF) showed an SMD of -0.50 (95\% CI, -0.61 to -0.40 ), indicating moderate effect in favor of participants receiving intervention compared to participants receiving control. A high between-study heterogeneity was observed, $\tau^{2}=0.07$, with substantial inconsistency across studies $\left(I^{2}=\right.$ $64 \%)$. However, the fixed-effect analysis was in agreement with the random-effect model, resulting in a pooled SMD of $-0.53(-0.60$ to -0.47$)$. The stratified meta-analyses for PF did not result in a significant reduction of $\tau^{2}$; type of intervention did not seem to be an important factor to the inconsistency observed across axSpA trials, when measuring change in PF.

\section{Pain}

In total, thirty trials (41 intervention comparisons, 4877 participants) were included in the analysis. Pooled analysis revealed statistically significant reduction in pain with an overall SMD of -0.48 (0.66 to -0.30$)$, indicating moderate effect across all interventions in axSpA trials. Between-study inconsistency was substantial $\left(I^{2}=62 \%\right)$. A large reduction in heterogeneity was found in the "type of intervention variable" [58] (i.e., non-pharmacological [58] vs. pharmacological $[\mathrm{P}])$, which in turn resulted in a significant reduction in $\tau^{2}$ at $32 \%$, supported by a statistically highly significant $P$ value $(P<0.001)$ for interaction between NP and $P$. Trials with pharmacological interventions had a pooled SMD of -0.64 $(-0.78$ to -0.49$)$, whereas trials with NP interventions had an overall SMD of 0.26 ( -0.20 to 0.72$)$.

\section{Spinal mobility}

Forty-three trials (45 comparisons, 5091 participants) were included in our meta-analysis. Pooled analysis revealed a small improvement in spinal mobility (SM) with an overall SMD of $-0.32(-0.48$ to -0.17$)$. A high between-study heterogeneity was observed, $\tau^{2}=0.21$, with a large inconsistency across studies $\left(I^{2}=83 \%\right)$. None of the subgroup analyses resulted in a significant reduction in $\tau^{2}$. 
Table 1 Study characteristics and risk of bias assessment of included studies

\begin{tabular}{|c|c|c|c|c|c|c|c|c|}
\hline Author & Year & $\begin{array}{l}\text { No. of patients } \\
\text { randomized }\end{array}$ & $\begin{array}{l}\text { No. of patients } \\
\text { receiving } \\
\text { intervention }\end{array}$ & $\begin{array}{l}\text { No. of patients } \\
\text { receiving comparison }\end{array}$ & $\begin{array}{l}\text { Trial duration } \\
\text { (weeks) }\end{array}$ & Intervention & Comparator & $\begin{array}{l}\text { Risk of bias* selection/ } \\
\text { performance/attrition/ } \\
\text { reporting }\end{array}$ \\
\hline Dougados & 1986 & 30 & 15 & 15 & 26 & SSZ (P) & $P L$ & $\mathrm{~A} / \mathrm{A} / \mathrm{A} / \mathrm{A}$ \\
\hline Feltelius & 1986 & 37 & 18 & 19 & 12 & SSZ (P) & $P L$ & U/U/A/A \\
\hline Nissila & 1988 & 85 & 43 & 42 & 26 & SSZ (P) & $P L$ & U/U/A/A \\
\hline Davis & 1989 & 30 & 15 & 15 & 12 & SSZ (P) & $P L$ & U/U/A/A \\
\hline Kraag & 1989 & 53 & 26 & 27 & 16 & $\begin{array}{l}\text { Supervised } \\
\text { training (NP) }\end{array}$ & SC & U/U/I/A \\
\hline Winkler & 1989 & 63 & 31 & 32 & 24 & SSZ (P) & $P L$ & U/U/U/A \\
\hline Corkill & 1990 & 62 & 32 & 30 & 48 & SSZ (P) & $P L$ & $\mathrm{~A} / \mathrm{A} / \mathrm{I} / \mathrm{A}$ \\
\hline Krajnc & 1990 & 95 & 71 & 24 & 24 & SSZ (P) & $P L$ & U/U/A/A \\
\hline Taylor & 1991 & 40 & 20 & 20 & 52 & SSZ (P) & $P L$ & $\mathrm{~A} / \mathrm{A} / \mathrm{A} / \mathrm{I}$ \\
\hline Hidding & 1993 & 144 & 68 & 76 & 36 & $\begin{array}{l}\text { Group } \\
\text { physiotherapy } \\
\text { (NP) }\end{array}$ & $A C$ & $\mathrm{~A} / \mathrm{A} / \mathrm{I} / \mathrm{A}$ \\
\hline Kirwan & 1993 & 89 & 44 & 45 & 156 & SSZ (P) & $P L$ & $\mathrm{~A} / \mathrm{A} / \mathrm{A} / \mathrm{I}$ \\
\hline \multirow[t]{4}{*}{ Dougados } & 1994 & 70 & 46 & 24 & 12 & $\begin{array}{l}\text { Ximoprofen } 5 \\
\mathrm{mg}(\mathrm{P})\end{array}$ & $P L$ & A/A/U/A \\
\hline & 1994 & 73 & 49 & 24 & 34 & $\begin{array}{l}\text { Ximoprofen } 10 \\
\mathrm{mg}(\mathrm{P})\end{array}$ & $P L$ & A/A/U/A \\
\hline & 1994 & 69 & 45 & 24 & 8 & $\begin{array}{l}\text { Ximoprofen } 20 \\
\mathrm{mg}(\mathrm{P})\end{array}$ & $P L$ & A/A/U/A \\
\hline & 1994 & 74 & 50 & 24 & 6 & $\begin{array}{l}\text { Ximoprofen } 30 \\
\text { mg (P) }\end{array}$ & $P L$ & A/A/U/A \\
\hline Clegg & 1996 & 264 & 131 & 133 & 36 & SSZ (P) & $P L$ & U/U/A/A \\
\hline \multirow[t]{2}{*}{ Helliwell } & 1996 & 22 & 15 & 7 & 44 & $\begin{array}{l}\text { In-patient } \\
\text { physiotherapy } \\
\text { (NP) }\end{array}$ & SC & I/I/I/A \\
\hline & 1996 & 22 & 15 & 7 & 44 & $\begin{array}{l}\text { Out-patient } \\
\text { hydrotherapy } \\
\text { (NP) }\end{array}$ & SC & $\mathrm{I} / \mathrm{I} / \mathrm{I} / \mathrm{A}$ \\
\hline \multirow[t]{3}{*}{ Dougados } & 1999 & 148 & 108 & 40 & 6 & Piroxicam (P) & $P L$ & U/U/A/A \\
\hline & 1999 & 160 & 120 & 40 & 6 & $\begin{array}{l}\text { Meloxicam } 15 \\
\mathrm{mg}(\mathrm{P})\end{array}$ & $P L$ & U/U/A/A \\
\hline & 1999 & 164 & 124 & 40 & 6 & $\begin{array}{l}\text { Meloxicam } 22.5 \\
\mathrm{mg}(\mathrm{P})\end{array}$ & $P L$ & U/U/A/A \\
\hline Altan & 2001 & 51 & 26 & 25 & 52 & MTX (P) & $A C$ & $\mathrm{U} / \mathrm{U} / \mathrm{I} / \mathrm{I}$ \\
\hline \multirow[t]{2}{*}{ Dougados } & 2001 & 118 & 80 & 38 & 6 & Celecoxib (P) & $P L$ & U/U/U/A \\
\hline & 2001 & 128 & 90 & 38 & 6 & Ketoprofen (P) & $\mathrm{PL}$ & U/U/U/A \\
\hline Van Tubergen & 2001 & 120 & 80 & 40 & 3 & $\begin{array}{l}\text { Spa-exercise } \\
\text { therapy (NP) }\end{array}$ & $A C$ & $\mathrm{~A} / \mathrm{A} / \mathrm{I} / \mathrm{A}$ \\
\hline Braun & 2002 & 70 & 35 & 35 & 12 & Infliximab (B) & $\mathrm{PL}$ & A/A/A/I \\
\hline Gorman & 2002 & 40 & 20 & 20 & 16 & Etanercept (B) & $P L$ & A/A/A/A \\
\hline Roychowdhury & 2002 & 30 & 14 & 16 & 24 & MTX (P) & $P L$ & U/U/A/A \\
\hline Schmidt & 2002 & 70 & 34 & 36 & 26 & SSZ (P) & $P L$ & U/U/U/A \\
\hline Sweeney & 2002 & 200 & 100 & 100 & 26 & $\begin{array}{l}\text { Supervised } \\
\text { training (NP) }\end{array}$ & SC & U/U/I/A \\
\hline Analay & 2003 & 51 & 27 & 24 & 12 & $\begin{array}{l}\text { Supervised } \\
\text { training (NP) }\end{array}$ & SC & $\mathrm{A} / \mathrm{A} / \mathrm{I} / \mathrm{A}$ \\
\hline Brandt & 2003 & 33 & 16 & 17 & 6 & Etanercept (B) & $P L$ & $\mathrm{~A} / \mathrm{A} / \mathrm{A} / \mathrm{I}$ \\
\hline
\end{tabular}


Table 1 Study characteristics and risk of bias assessment of included studies (Continued)

\begin{tabular}{|c|c|c|c|c|c|c|c|c|}
\hline Author & Year & $\begin{array}{l}\text { No. of patients } \\
\text { randomized }\end{array}$ & $\begin{array}{l}\text { No. of patients } \\
\text { receiving } \\
\text { intervention }\end{array}$ & $\begin{array}{l}\text { No. of patients } \\
\text { receiving comparison }\end{array}$ & $\begin{array}{l}\text { Trial duration } \\
\text { (weeks) }\end{array}$ & Intervention & Comparator & $\begin{array}{l}\text { Risk of bias* selection/ } \\
\text { performance/attrition/ } \\
\text { reporting }\end{array}$ \\
\hline Davis & 2003 & 277 & 138 & 139 & 24 & Etanercept (B) & $\mathrm{PL}$ & A/A/A/A \\
\hline Calin & 2004 & 84 & 45 & 39 & 12 & Etanercept (B) & $P L$ & U/U/A/A \\
\hline $\begin{array}{l}\text { Gonzalez- } \\
\text { Lopez }\end{array}$ & 2004 & 35 & 17 & 18 & 24 & MTX (P) & $P L$ & A/A/A/I \\
\hline Codish & 2005 & 28 & 14 & 14 & 12 & $\begin{array}{l}\text { Balneo therapy } \\
\text { (NP) }\end{array}$ & $A C$ & U/U/I/A \\
\hline D'Las Penas & 2005 & 40 & 20 & 20 & 16 & $\begin{array}{l}\text { Supervised } \\
\text { training (NP) }\end{array}$ & SC & $\mathrm{A} / \mathrm{A} / \mathrm{I} / \mathrm{A}$ \\
\hline Lim & 2005 & 50 & 25 & 25 & 8 & $\begin{array}{l}\text { Supervised } \\
\text { training (NP) }\end{array}$ & SC & $\mathrm{U} / \mathrm{U} / \mathrm{I} / \mathrm{A}$ \\
\hline Marzo-Ortega & 2005 & 42 & 28 & 14 & 30 & Infliximab (B) & $P L$ & $\mathrm{~A} / \mathrm{A} / \mathrm{U} / \mathrm{A}$ \\
\hline Van der Heijde & 2005 & 279 & 201 & 78 & 24 & Infliximab (B) & $P L$ & U/U/A/A \\
\hline \multirow[t]{3}{*}{ Van der Heijde } & 2005 & 134 & 103 & 31 & 6 & Etoricoxib (P) & $P L$ & A/A/A/A \\
\hline & 2005 & 123 & 92 & 31 & 6 & Etoricoxib (P) & $P L$ & A/A/A/A \\
\hline & 2005 & 130 & 99 & 31 & 6 & Naproxen (P) & $P L$ & A/A/A/A \\
\hline Altan & 2006 & 60 & 30 & 30 & 24 & $\begin{array}{l}\text { Balneo therapy } \\
\text { (NP) }\end{array}$ & $A C$ & U/U/I/A \\
\hline \multirow[t]{3}{*}{ Barkhuizen } & 2006 & 189 & 137 & 52 & 12 & $\begin{array}{l}\text { Celecoxib } 200 \\
\text { mg (P) }\end{array}$ & $P L$ & U/U/U/A \\
\hline & 2006 & 213 & 161 & 52 & 12 & $\begin{array}{l}\text { Celecoxib } 400 \\
\text { mg (P) }\end{array}$ & $P L$ & U/U/U/A \\
\hline & 2006 & 209 & 157 & 52 & 12 & Naproxen (P) & $P L$ & U/U/U/A \\
\hline Ince & 2006 & 30 & 15 & 15 & 12 & $\begin{array}{l}\text { Supervised } \\
\text { training (NP) }\end{array}$ & SC & U/U/I/A \\
\hline Van der Heijde & 2006 & 315 & 208 & 107 & 24 & Adalimumab (B) & $P L$ & $\mathrm{~A} / \mathrm{A} / \mathrm{A} / \mathrm{A}$ \\
\hline \multirow[t]{2}{*}{ Van der Heijde } & 2006 & 180 & 155 & 25 & 24 & Etanercept (B) & $P L$ & U/U/U/A \\
\hline & 2006 & 175 & 150 & 25 & 24 & Etanercept (B) & $P L$ & U/U/U/A \\
\hline Lambert & 2007 & 82 & 38 & 44 & 24 & Adalimumab (B) & $P L$ & U/U/A/A \\
\hline Huang & 2008 & 126 & 83 & 43 & 8 & Etanercept (B) & $P L$ & U/U/U/U \\
\hline \multirow[t]{2}{*}{ Inman } & 2008 & 177 & 138 & 39 & 14 & Golimumab (B) & $P L$ & $\mathrm{~A} / \mathrm{A} / \mathrm{A} / \mathrm{A}$ \\
\hline & 2008 & 179 & 149 & 39 & 14 & Golimumab (B) & $P L$ & $\mathrm{~A} / \mathrm{A} / \mathrm{A} / \mathrm{A}$ \\
\hline Barkham & 2010 & 40 & 20 & 20 & 12 & Etanercept (B) & $P L$ & U/U/A/A \\
\hline Inman & 2010 & 76 & 39 & 37 & 12 & Infliximab (B) & $P L$ & U/U/U/A \\
\hline Braun & 2011 & 566 & 379 & 187 & 16 & Etanercept (B) & $A C$ & A/A/A/A \\
\hline Dougados & 2011 & 82 & 39 & 43 & 12 & Etanercept (B) & $P L$ & U/U/A/I \\
\hline $\begin{array}{l}\text { Navarro- } \\
\text { Sarabia }\end{array}$ & 2011 & 108 & 54 & 54 & 12 & Etanercept (B) & $A C$ & $\mathrm{~A} / \mathrm{A} / \mathrm{A} / \mathrm{A}$ \\
\hline $\mathrm{Hu}$ & 2012 & 46 & 26 & 20 & 12 & Adalimumab (B) & $P L$ & U/U/U/I \\
\hline Bao & 2014 & 213 & 108 & 105 & 24 & Golimumab (B) & $P L$ & U/U/U/A \\
\hline Huang & 2014 & 344 & 229 & 114 & 12 & Adalimumab (B) & $P L$ & $\mathrm{~A} / \mathrm{A} / \mathrm{A} / \mathrm{A}$ \\
\hline
\end{tabular}

*Shown as selection bias (methods for sequence generation and allocation)/performance bias (blinding of participants and personnel)/attrition bias (incomplete outcome data)/reporting bias (selective outcome reporting). Abbreviations: SSZ sulfasalazine, $P$ pharmacological modalities, $P L$ placebo, NP non-pharmacological modalities, $B$ biological modalities, SC standard care, $A C$ active comparison, $A$ adequate, $U$ unclear, $I$ inadequate

\section{Spinal stiffness}

In total, 25 trials (34 comparisons, 3658 participants) were included in the analysis. The overall effect size revealed substantive statistically significant improvement in spinal stiffness, SMD of -0.59 (0.74 to -0.44$)$, indicating moderate effect of all interventions in axSpA trials. The heterogeneity was large, $\quad \tau^{2}=0.14$, with substantial inconsistency 
Table 2 Outcome matrix

\begin{tabular}{|c|c|c|c|c|c|c|c|c|c|}
\hline Author & Physical function & Pain & Spinal mobility & Spinal stiffness & $\begin{array}{l}\text { Patient's global } \\
\text { assessment }\end{array}$ & $\begin{array}{l}\text { Peripheral } \\
\text { joints/entheses }\end{array}$ & $\begin{array}{l}\text { Acute phase } \\
\text { reactants }\end{array}$ & $\begin{array}{l}\text { Spine/hip } \\
\text { radiograph }\end{array}$ & Fatigue \\
\hline \multicolumn{10}{|l|}{ SM-ARD trials } \\
\hline $\begin{array}{l}\text { Dougados (1994) } \\
\text { [41] }\end{array}$ &,$++(\mathrm{DFI})$ & $\begin{array}{l}+,+ \\
\text { (VAS) }\end{array}$ &,++ (Schober) &,++ (minutes) & - & - & - & $-/-$ & - \\
\hline $\begin{array}{l}\text { Dougados (1999) } \\
\text { [42] }\end{array}$ &,$++(\mathrm{DFl})$ & $\begin{array}{l}+,+ \\
\text { (VAS) }\end{array}$ &,++ (Schober) &,++ (minutes) &,++ (VAS) & - &,$++(\mathrm{CRP})$ & $-1-$ & - \\
\hline $\begin{array}{l}\text { Dougados (2001) } \\
\text { [43] }\end{array}$ &,$++($ BASFI) & $\begin{array}{l}+,+ \\
\text { (VAS) }\end{array}$ &,++ (Schober) &,++ (minutes) &,++ (VAS) & - &,$++(\mathrm{CRP})$ & $-/-$ & - \\
\hline $\begin{array}{l}\text { Van der Heijde } \\
\text { (2005_2) [44] }\end{array}$ &,$++($ BASFI) & $\begin{array}{l}+,+ \\
\text { (VAS) }\end{array}$ &,++ (Schober) &,++ (minutes) &,++ (VAS) & $\begin{array}{l}+,+ \\
\text { (BASDAl question 4) }\end{array}$ &,$++(\mathrm{CRP})$ & $-/-$ & - \\
\hline $\begin{array}{l}\text { Barkhuizen (2006) } \\
\text { [27] }\end{array}$ &,$++($ BASFI) & $\begin{array}{l}+,+ \\
\text { (VAS) }\end{array}$ &,++ (Schober) &,++ (minutes) &,++ (VAS) & - &,$++(\mathrm{CRP})$ & $-/-$ & - \\
\hline \multicolumn{10}{|c|}{ Physical therapy trials } \\
\hline Kraag (1990) [45] &,$++($ TADLQ $)$ & $\begin{array}{l}+,+ \\
\text { (VAS) }\end{array}$ &,++ (Schober) &,$++($ TADLQ $)$ & - & - & - & $-1-$ & - \\
\hline $\begin{array}{l}\text { Hidding (1993) } \\
\text { [46] }\end{array}$ &,$++(\mathrm{DFI} 0)$ & $\begin{array}{l}+,+ \\
\text { (VAS) }\end{array}$ &,++ (Schober) &,++ (VAS) &,++ (VAS) & $\begin{array}{l}+,+ \\
\text { (enthesitis index) }\end{array}$ & - & $-/-$ & - \\
\hline Helliwell [31] & - & $\begin{array}{l}+,+/- \\
\text { (VAS) }\end{array}$ &,++ (Schober) &,$++/-($ VAS) & - & - & - & $-/-$ & - \\
\hline $\begin{array}{l}\text { Van Tubergen } \\
\text { (2001) [47] }\end{array}$ &,$++($ BASFI) & $\begin{array}{l}+,+ \\
\text { (VAS) }\end{array}$ & - &,++ (minutes) &,++ (VAS) & - & - & $-1-$ & - \\
\hline $\begin{array}{l}\text { Sweeney (2002) } \\
\text { [48] }\end{array}$ &,$++($ BASFI) & $\begin{array}{l}+,+ \\
\text { (SES) }\end{array}$ & - & - &,$++($ BAS-G) & - & - & $-/-$ & - \\
\hline Analay (2003) [49] &,$++($ BASFI) & $\begin{array}{l}+,+ \\
\text { (VAS) }\end{array}$ &,++ (Schober) &,++ (minutes) & - & - & - & $-1-$ & - \\
\hline $\begin{array}{l}\text { Codish (2005) } \\
{[50]}\end{array}$ &,$++($ BASFI) & $\begin{array}{l}+,+ \\
\text { (VAS) }\end{array}$ & $\begin{array}{l}+,+/- \\
\text { (Schober) }\end{array}$ & - & - & - & - & $-/-$ & - \\
\hline $\begin{array}{l}\text { Fernandez-de- } \\
\text { Las-Penas (2005) } \\
\text { [51] }\end{array}$ &,$++(\mathrm{BASFI})$ & - &,++ (Schober) & - & - & - & - & $-1-$ & - \\
\hline Lim [30] &,$++($ BASFI) & $\begin{array}{l}+,+/- \\
\text { (VAS) }\end{array}$ &,$++($ FFD) & - & - & - & - & $-/-$ & - \\
\hline Ince (2006) [52] & - & - &,++ (Schober) & - & - & - & - & $-1-$ & - \\
\hline Altan (2006) [53] &,$++($ BASFI) & $\begin{array}{l}+,+ \\
\text { (VAS) }\end{array}$ &,++ (Schober) &,++ (NRS) &,$++(\mathrm{NRS})$ & - & - & $-/-$ & - \\
\hline \multicolumn{10}{|l|}{ DC-ART trials } \\
\hline $\begin{array}{l}\text { Dougados (1986) } \\
\text { [54] }\end{array}$ &,$++(\mathrm{DFI})$ & $\begin{array}{l}+,+ \\
\text { (VAS) }\end{array}$ &,++ (Schober) &,++ (minutes) & - & $\begin{array}{l}+,+ \\
\text { (enthesitis index) }\end{array}$ &,$++(E S R)$ & $-1-$ & - \\
\hline Feltelius [28] & - & $\begin{array}{l}+,+/- \\
\text { (VAS) }\end{array}$ & $\begin{array}{l}+,+/- \\
\text { (Schober) }\end{array}$ &,++ (VAS) &,$++(V A S)$ & $\begin{array}{l}+,+ \\
\text { (enthesitis index) }\end{array}$ &,$++(E S R)$ & $-/-$ & - \\
\hline Nissila (1988) [55] & - & $\begin{array}{l}+,+ \\
\text { (VAS) }\end{array}$ &,++ (Schober) &,++ (minutes) &,++ (VAS) &,++ (22-joint count) &,$++(\mathrm{CRP})$ & $-/-$ & - \\
\hline Davis (1989) [56] & - & $\begin{array}{l}+,+ \\
\text { (VAS) }\end{array}$ & $\begin{array}{l}+,+ \text { (occiput-to- } \\
\text { wall distance) }\end{array}$ &,$++($ VAS $)$ & - & - &,$++(\mathrm{CRP})$ & $-/-$ & - \\
\hline $\begin{array}{l}\text { Winkler (1989) } \\
\text { [57] }\end{array}$ & - & $\begin{array}{l}+,+ \\
(\text { VAS) }\end{array}$ &,++ (Schober) &,++ (hours) &,++ (VAS) &,++ (66-joint count) &,$++(E S R)$ & $-/-$ & - \\
\hline Corkill [58] & - & $\begin{array}{l}+,+ \\
\text { (VAS) }\end{array}$ & $\begin{array}{l}+,+ \text { (occiput-to- } \\
\text { wall distance) }\end{array}$ &,$++($ VAS $)$ & - & - &,$++(\mathrm{CRP})$ & $-1-$ & - \\
\hline Krajnc (1990) [59] & - & - &,++ (Schober) & & - & - & - & $-/-$ & - \\
\hline Taylor [34] & - & $\begin{array}{l}+,+ \\
(\text { VAS) }\end{array}$ &,++ (Schober) &,++ (VAS) &,$++/-(\mathrm{NRS})$ &,$++/-$ (joint count) &,$++($ CRP) & $\begin{array}{l}+,+/- \text { SIJ } \\
\text { score }\end{array}$ & - \\
\hline Kirwan [29] &,$+-(\mathrm{HAQ})$ & $\begin{array}{l}+,- \\
\text { (VAS) }\end{array}$ &,++ (Schober) &,$++/-($ VAS) &,$+-(V A S)$ & $\begin{array}{l}+,+/- \\
\text { (44-joint count) }\end{array}$ & - & $-1-$ & - \\
\hline Clegg (1996) [60] &,$++(\mathrm{DFl})$ & $\begin{array}{l}+,+ \\
\text { (VAS) }\end{array}$ &,++ (Schober) &,++ (minutes) &,$++/-(\mathrm{NRS})$ &,$++(44$-joint count) &,$++(\mathrm{CRP})$ & $-1-$ & - \\
\hline
\end{tabular}


Table 2 Outcome matrix (Continued)

\begin{tabular}{|c|c|c|c|c|c|c|c|c|c|}
\hline Author & Physical function & Pain & Spinal mobility & Spinal stiffness & $\begin{array}{l}\text { Patient's global } \\
\text { assessment }\end{array}$ & $\begin{array}{l}\text { Peripheral } \\
\text { joints/entheses }\end{array}$ & $\begin{array}{l}\text { Acute phase } \\
\text { reactants }\end{array}$ & $\begin{array}{l}\text { Spine/hip } \\
\text { radiograph }\end{array}$ & Fatigue \\
\hline Altan [35] &,++ (DFI) & $\begin{array}{l}+,+ \\
\text { (VAS) }\end{array}$ &,+- (Schober) &,++ (minutes) &,++ (NRS) & $\begin{array}{l}+,++ \text { (enthesitis } \\
\text { index) }\end{array}$ &,$++($ CRP) & $-1-$ & - \\
\hline Braun [36] &,$++($ BASFI) & $\begin{array}{l}+,+/- \\
\text { (NRS) }\end{array}$ &,$++($ BASMI) & $\begin{array}{l}+,+/- \\
\text { (minutes) }\end{array}$ &,$++(\mathrm{NRS})$ &,$++(44$-joint count $)$ &,$++(\mathrm{CRP})$ & $-1-$ & $\begin{array}{l}+,+/- \\
\text { (NRS) }\end{array}$ \\
\hline $\begin{array}{l}\text { Gorman (2002) } \\
{[61]}\end{array}$ &,$++($ BASFI) & $\begin{array}{l}+,+/- \\
\text { (VAS) }\end{array}$ &,++ (Schober) &,++ (minutes) &,++ (VAS) &,$++(66$-joint count $)$ &,$++(\mathrm{CRP})$ & $-1-$ & - \\
\hline $\begin{array}{l}\text { Roychowdhury } \\
\text { (2002) [62] }\end{array}$ & - & - &,$++($ BASMI) &,++ (minutes) & - & - &,$++(\mathrm{CRP})$ & $-/-$ & - \\
\hline $\begin{array}{l}\text { Schmidt (2002) } \\
\text { [63] }\end{array}$ &,$++(\mathrm{DFl})$ & $\begin{array}{l}+,+ \\
\text { (VAS) }\end{array}$ &,++ (Schober) &,++ (minutes) & - & $\begin{array}{l}+,+ \\
\text { (enthesitis index) }\end{array}$ &,$++(\mathrm{CRP})$ & $-1-$ & - \\
\hline Brandt [37] &,$++($ BASFI) & $\begin{array}{l}+,+/- \\
\text { (NRS) }\end{array}$ &,$++($ BASMI) &,$++/-(\mathrm{NRS})$ & - & $\begin{array}{l}+,+/- \\
\text { (66-joint count) }\end{array}$ &,$++/-(\mathrm{CRP})$ & $-1-$ & $\begin{array}{l}+,+/- \\
\text { (NRS) }\end{array}$ \\
\hline Davis (2003) [64] &,$++($ BASFI) & $\begin{array}{l}+,+/- \\
\text { (VAS) }\end{array}$ &,++ (Schober) &,++ (minutes) &,++ (VAS) &,$+--(68$-joint count) &,$++(\mathrm{CRP})$ & $-1-$ & - \\
\hline Calin (2004) [65] &,$++($ BASFI 0) & $\begin{array}{l}+,+ \\
\text { (VAS) }\end{array}$ &,$++($ BASMI) &,++ (minutes) &,++ (VAS) &,$+-(68$-joint count) &,$++(\mathrm{CRP})$ & $-1-$ &,$++($ VAS $)$ \\
\hline $\begin{array}{l}\text { Gonzalez-Lopez } \\
\text { [38] }\end{array}$ &,$++($ BASFI) & $\begin{array}{l}+,+ \\
\text { (VAS) }\end{array}$ & - &,++ (VAS) &,$++($ VAS $)$ &,++ (44-joint count) &,$+-(E S R)$ & $-1-$ & - \\
\hline $\begin{array}{l}\text { Marzo-Ortega } \\
\text { (2005) [66] }\end{array}$ &,$++($ BASFI) & $\begin{array}{l}+,+ \\
\text { (VAS) }\end{array}$ & - &,++ (minutes) & - & $\begin{array}{l}+,+ \\
\text { (enthesitis index) }\end{array}$ &,$+-(E S R)$ & $-1-$ & - \\
\hline $\begin{array}{l}\text { Van der Heijde } \\
\text { (2005_1) [44] }\end{array}$ &,$++($ BASFI) & $\begin{array}{l}+,+ \\
\text { (VAS) }\end{array}$ &,$++($ BASMI) & - &,++ (VAS) & $\begin{array}{l}+,+ \\
\text { (enthesitis Index) }\end{array}$ &,$++(\mathrm{CRP})$ & $-1-$ & - \\
\hline $\begin{array}{l}\text { Van der Heijde } \\
\text { [32] }\end{array}$ &,$++($ BASFI) & $\begin{array}{l}+,+ \\
\text { (VAS) }\end{array}$ &,$++($ BASMI) &,++ (VAS) &,++ (VAS) &,$++(44$-joint count $)$ &,$++(\mathrm{CRP})$ & $-1-$ & - \\
\hline $\begin{array}{l}\text { Van der Heijde } \\
\text { (2006_2) [67] }\end{array}$ &,$++($ BASFI) & $\begin{array}{l}+,+ \\
\text { (VAS) }\end{array}$ &,++ (Schober) &,++ (VAS) &,$++($ VAS $)$ &,++ (70-joint count) &,$++(\mathrm{CRP})$ & $-/-$ & - \\
\hline $\begin{array}{l}\text { Lambert (2007) } \\
\text { [68] }\end{array}$ &,$++(\mathrm{BASFI})$ & $\begin{array}{l}+,+ \\
\text { (VAS) }\end{array}$ &,$++($ BASMI) &,$++(\mathrm{VAS})$ &,++ (VAS) &,$++(44$-joint count $)$ &,$++(\mathrm{CRP})$ & $-1-$ & - \\
\hline Inman (2008) [69] &,$++($ BASFI) & $\begin{array}{l}+,+ \\
\text { (VAS) }\end{array}$ &,$++($ BASMI) &,$++(V A S)$ &,++ (VAS) & - &,$++(\mathrm{CRP})$ & $-1-$ & $\begin{array}{l}+,+ \\
(J E S Q)\end{array}$ \\
\hline $\begin{array}{l}\text { Barkham (2010) } \\
\text { [70] }\end{array}$ &,$++($ BASFI) & - & - &,++ (VAS) & - & - & - & $-1-$ & - \\
\hline Inman (2010) [71] &,$++($ BASFI) & - &,$++($ BASMI) & - &,$++($ BAS-G) & - &,$++(\mathrm{CRP})$ & $-1-$ & - \\
\hline Braun (2011) [72] &,$++($ BASFI) & $\begin{array}{l}+,+ \\
\text { (VAS) }\end{array}$ &,$++($ BASMI) &,$++/-($ VAS) &,++ (VAS) &,$++(66$-joint count $)$ &,$++(\mathrm{CRP})$ & $-/-$ & - \\
\hline Dougados [39] &,$++($ BASFI) & $\begin{array}{l}+,+ \\
\text { (VAS) }\end{array}$ &,$++($ BASMI) & - &,$+-(V A S)$ & - &,$++(\mathrm{CRP})$ & $-/-$ & - \\
\hline $\begin{array}{l}\text { Navarro-Sarabia } \\
\text { (2011) [73] }\end{array}$ &,$++($ BASFI) & $\begin{array}{l}+,+ \\
\text { (VAS) }\end{array}$ &,$++($ BASMI) & - &,++ (VAS) &,$++(66$-joint count) &,$++(\mathrm{CRP})$ & $-1-$ & $\begin{array}{l}+,+/- \\
\text { (VAS) }\end{array}$ \\
\hline $\mathrm{Hu}[40]$ &,$++($ BASFI) & $\begin{array}{l}+,+ \\
\text { (VAS) }\end{array}$ & - & - & - & - &,$++(\mathrm{CRP})$ & $-1-$ & $\begin{array}{l}+,+/- \\
\text { (VAS 0- } \\
10)\end{array}$ \\
\hline Bao (2014) [74] &,$++(\mathrm{BASFI})$ & $\begin{array}{l}+,+ \\
\text { (VAS) }\end{array}$ &,$++($ BASMI) & - & - & - &,$++(\mathrm{CRP})$ & $-/-$ & $\begin{array}{l}+,+ \\
(J E S Q)\end{array}$ \\
\hline Huang (2014) [75] &,$++(\mathrm{BASFI})$ & $\begin{array}{l}+,+ \\
\text { (VAS) }\end{array}$ &,$++($ BASMI) &,++ (VAS) &,++ (VAS) &,$++(44$-joint count $)$ &,++ (CRP) & $-1-$ & - \\
\hline
\end{tabular}

,++ indicates that outcome was measured and fully reported

,$++/-$ indicates that outcome was measured and partially reported (e.g., only the $P$ value is given for the comparison)

,+- indicates that outcome was measured but not reported

- indicates that outcome was not measured

Abbreviations: DFI Dougados functional index, VAS visual analogue scale, NRS numeric range scale, ESR erythrocyte sedimentation rate, CRP C-reactive

protein, BASFI Bath Ankylosing Spondylitis Functional Index, TADLQ Toronto Activity of Daily Living Questionnaire, HAQ Health Assessment

Questionnaire, BASRI Bath Ankylosing Spondylitis Radiology Index, SES Stanford Self-Efficacy Scale, BAS-G Bath Ankylosing Spondylitis Global Index,

BASDAI Bath Ankylosing Disease Activity Index, SPARCC SpondyloArthritis Research Consortium of Canada, JESQ Jenkins Sleep Evaluation Questionnaire 
Table 3 Results of the stratified meta-analyses

\begin{tabular}{|c|c|c|c|c|c|c|}
\hline Variable & No. of trials/comparisons & SMD & $95 \% \mathrm{Cl}$ & $R^{2}$ & $\mathrm{Tau}^{2}$ & $P$ for interaction \\
\hline All trials, physical function & $33 / 43$ & -0.50 & $-0.61,-0.40$ & $64 \%$ & 0.07 & - \\
\hline Fixed-effect model & & -0.53 & $-0.57,-0.45$ & & & \\
\hline Intervention & & & & & 0.08 & 0.42 \\
\hline Pharmacological & & -0.53 & $-0.64,-0.42$ & & & \\
\hline Non-pharmacological & & -0.40 & $-0.74,-0.05$ & & & \\
\hline Intervention & & & & & 0.08 & 0.44 \\
\hline Biological treatment & & -0.57 & $-0.68,-0.46$ & & & \\
\hline Other treatment & & -0.46 & $-0.63,-0.28$ & & & \\
\hline All trials, pain & $30 / 41$ & -0.48 & $-0.66,-0.30$ & $87 \%$ & 0.28 & - \\
\hline Fixed-effect model & & -0.50 & $-0.56,-0.44$ & & & \\
\hline Intervention & & & & & 0.19 & $<0.001$ \\
\hline Pharmacological & & -0.64 & $-0.78,-0.49$ & & & \\
\hline Non-pharmacological & & 0.26 & $-0.20,0.72$ & & & \\
\hline Intervention & & & & & 0.29 & 0.28 \\
\hline Biological treatment & & -0.64 & $-0.80,-0-49$ & & & \\
\hline Other treatment & & -0.41 & $-0.68,-0.14$ & & & \\
\hline All trials, spinal mobility & $30 / 45$ & -0.32 & $-0.48,-0.17$ & $83 \%$ & 0.21 & - \\
\hline Fixed-effect model & & -0.32 & $-0.38,-0.26$ & & & \\
\hline Intervention & & & & & 0.19 & 0.28 \\
\hline Pharmacological & & -0.35 & $-0.51,-0.19$ & & & \\
\hline Non-pharmacological & & -0.02 & $-0.61,0.56$ & & & \\
\hline Intervention & & & & & 0.33 & 0.65 \\
\hline Biological treatment & & -0.31 & $-0.47,-0.15$ & & & \\
\hline Other treatment & & -0.28 & $-0.52,-0.03$ & & & \\
\hline All trials, spinal stiffness & $25 / 34$ & -0.59 & $-0.74,-0.44$ & $75 \%$ & 0.14 & - \\
\hline Fixed-effect model & & -0.61 & $-0.68,-0.54$ & & & \\
\hline Intervention & & & & & 0.18 & 0.95 \\
\hline Pharmacological & & -0.60 & $-0.76,-0.44$ & & & \\
\hline Non-pharmacological & & -0.61 & $-0.93,-0.29$ & & & \\
\hline Intervention & & & & & 0.16 & 0.34 \\
\hline Biological treatment & & -0.77 & $-0.88,-0.65$ & & & \\
\hline Other treatment & & -0.55 & $-0.75,-0.35$ & & & \\
\hline All trials, PGA & $21 / 28$ & -0.71 & $-0.89,-0.54$ & $83 \%$ & 0.18 & - \\
\hline Fixed-effect model & & -0.74 & $-0.81,-0.67$ & & & \\
\hline Intervention & & & & & 0.19 & 0.15 \\
\hline Pharmacological & & -0.77 & $-0.96,-0.59$ & & & \\
\hline Non-pharmacological & & -0.37 & $-0.81,0.07$ & & & \\
\hline \multicolumn{7}{|l|}{ Intervention } \\
\hline Biological treatment & & -0.84 & $-1.09,-0.60$ & & 0.20 & 0.23 \\
\hline Other treatment & & -0.60 & $-0.87,-0.34$ & & & \\
\hline All trials, $\mathrm{PJ} / \mathrm{E}$ & $15 / 15$ & 0.05 & $-0.11,0.22$ & $68 \%$ & 0.06 & - \\
\hline Fixed-effect model & & 0.00 & $-0.08,0.09$ & & & \\
\hline Intervention & & & & & 0.00 & $<0.001$ \\
\hline Pharmacological & & -0.06 & $-0.15,0.03$ & & & \\
\hline
\end{tabular}


Table 3 Results of the stratified meta-analyses (Continued)

\begin{tabular}{|c|c|c|c|c|c|c|}
\hline$\underline{\text { Variable }}$ & No. of trials/comparisons & SMD & $95 \% \mathrm{Cl}$ & $P^{2}$ & $\mathrm{Tau}^{2}$ & $P$ for interaction \\
\hline Non-pharmacological & & 0.99 & $0.63,1.35$ & & & \\
\hline Intervention & & & & & 0.04 & 0.02 \\
\hline Biological treatment & & -0.10 & $-0.20,-0.01$ & & & \\
\hline Other treatment & & 0.27 & $-0.10,0.64$ & & & \\
\hline All trials, acute phase reactants & $27 / 31$ & -0.51 & $-0.70,-0.32$ & $84 \%$ & 0.22 & - \\
\hline Fixed-effect model & & -0.62 & $-0.69,-0.55$ & & & \\
\hline Intervention & & & & & - & - \\
\hline Pharmacological & & -0.51 & $-0.70,-0.32$ & & & \\
\hline Non-pharmacological & & - & - & & & \\
\hline Intervention & & & & & 0.12 & 0.001 \\
\hline Biological treatment & & -0.77 & $-1.02,-0.52$ & & & \\
\hline Other treatment & & -0.22 & $-0.37,-0.07$ & & -0.07 & \\
\hline All trials, spine radiographs & $1 / 1$ & 0.96 & $0.22,1.69$ & - & - & - \\
\hline Fixed-effect model & & - & - & & & \\
\hline Intervention & & & & & - & - \\
\hline Pharmacological & & - & - & & & \\
\hline Non-pharmacological & & - & - & & & \\
\hline \multicolumn{7}{|l|}{ Intervention } \\
\hline \multicolumn{7}{|l|}{ Biological treatment } \\
\hline Other treatment & & 0.96 & $0.22,1.69$ & & & \\
\hline All trials, fatigue & $3 / 4$ & -0.65 & $-0.82,-0.48$ & $0 \%$ & 0.00 & - \\
\hline Fixed-effect model & & -0.65 & $-0.82,-0.48$ & & & \\
\hline Intervention & & & & & - & - \\
\hline Pharmacological & & -0.65 & $-0.82,-0.48$ & & & \\
\hline Non-pharmacological & & - & - & & & \\
\hline Intervention & & & & & - & - \\
\hline Biological treatment & & -0.65 & $-0.82,-0.48$ & & & \\
\hline Other treatment & & & & & & \\
\hline
\end{tabular}

SMD standardized mean difference, $\mathrm{Cl}$ confidence interval, $l^{2}$ inconsistency (i.e., the percentage of total variation across studies due to heterogeneity), Tau ${ }^{2}$ tau squared is an estimate of the variance of the true effect sizes, $P J / E$ peripheral joint count/enthesitis index

between studies $\left(I^{2}=75 \%\right)$. The pre-specified stratified analyses did not result in a significant reduction of $\tau^{2}$, and type of intervention did not seem to be an important factor to the inconsistency observed across trials when measuring change in SS in axSpA trials.

\section{Patient's global assessment}

Twenty-one RCTs reported sufficient data to be included in the meta-analysis (28 comparisons, 4031 participants). A significantly pooled moderate effect favoring intervention with large inconsistency was observed, SMD $-0.71(-0.89$ to -0.54$)$ and $I^{2}=83 \%$. Stratified analyses did result in a significant reduction of $\tau^{2}$; the type of intervention did not seem to be an important factor to the inconsistency observed across trials when measuring change in PGA in axSpA trials.

\section{Peripheral joints and enthesitis index}

Fifteen trials (2334 participants) were included in our meta-analysis. A high between-study heterogeneity was observed, $\tau^{2}=0.05$, with a substantial inconsistency across studies $\left(I^{2}=68 \%\right)$ [13]. There was no significant difference in the joint count/enthesitis index after the interventions; the overall SMD was 0.05 ( -0.11 to 0.22$)$. A large reduction in heterogeneity was found in the "type of intervention variables" (i.e., NP vs. P treatments and B vs. other treatment $[\mathrm{O}])$, which in turn resulted in significant reductions in $\tau^{2}$, supported by statistically significant $P$ values for interactions between $\mathrm{NP} / \mathrm{P}$ and $\mathrm{B} / \mathrm{O}$ $(P<0.001$ and $P=0.03$, respectively). Treatment with a 
biological agent had a small effect on reducing the number of swollen joints in axSpA patients, SMD - 0.10 (0.20 to -0.01 ).

\section{Acute phase reactants}

Twenty-seven trials (31 comparisons, 3869 participants) were included in our meta-analysis. The overall analysis of change in APRs showed a moderate all-in-all effect for all interventions in axSpA trials, $\mathrm{SMD}=-0.51(-$ 0.70 to -0.32$)$, with a large inconsistency $\left(I^{2}=84 \%\right)$. A large reduction in heterogeneity was found in the "type of intervention variable" (i.e., B vs. O treatments), which in turn resulted in a significant reduction in $\tau^{2}$ at $45 \%$, supported by a statistically significant $P$ value $(P=0.001)$ for interaction between $\mathrm{B}$ and $\mathrm{O}$ treatments. Trials with a biological intervention had a large effect on reducing APRs, SMD of -0.77 (-1.02 to - 0.52), whereas trials with other treatment interventions (i.e., NSAIDs, MTX, SSZ, and NP) had an overall small effect, $\mathrm{SMD}=-0.22$ $(-0.37$ to -0.07$)$.

\section{Spine/hip radiographs}

Only two of the included trials reported a change in spine radiographs (SR). One trial reported insufficient data to be included in the meta-analysis. In total, only one trial with 32 axSpA patients was included in the analysis. The effect size was 0.96 (0.22 to 1.6), indicating SSZ did not have an effect on preventing spinal progression. No trial reported hip radiographs.

\section{Fatigue}

Three studies reported sufficient data to be included in the meta-analysis (4 comparisons, 653 participants). The overall SMD was -0.65 ( -0.82 to -0.48$)$, and no between-study inconsistency was found $\left(I^{2}=0 \%\right)$. The pre-specified stratified analyses performed with regression models did not influence the variation in the estimates across strata and were not considered relevant.

\section{Association with primary endpoint}

Overall, 27 trials (39 comparisons) stated explicitly what the primary endpoint measure was and reported the proportion of participants achieving the primary endpoint. The most commonly composite primary outcome was the ASAS 20 response criteria (56\%) followed by the change in BASDAI (37\%). Two studies (7\%) used a customized composite outcome (e.g., the overall change in PGA).

In total, 5723 axSpA patients were included in the meta-analysis. The pooled OR for achieving primary endpoint was 3.26 (2.58 to 4.13) in favor of participants receiving experimental intervention compared to participants receiving a control comparator.

Univariate meta-regression analyses based on all trials (i.e., trials that had a measured composite primary endpoint) indicated that a reduction in pain and APRs and improvements in PF and PGA were significantly associated with increased odds for achieving primary endpoint, whereas SM, SS, PJ/E, and fatigue were not (Table 4). We repeated the meta-regression analysis based on trials reporting all four domains significantly affecting the OR for achieving primary endpoint. PF, pain, and PGA were still significantly associated with the OR for achieving primary endpoint, whereas APRs proved non-significant. Multivariable meta-regression analyses showed that PF did not have a statistically significant explanatory effect on achieving primary outcome when the following explanatory core outcome domains pain, PGA, and APRs were added to the model simultaneously. Only reduction in pain and PGA had a statistically significant effect on

Table 4 Overview of the impact of core outcome domains on the odds ratio (OR) for achieving primary endpoint per trial

\begin{tabular}{|c|c|c|c|c|c|c|c|}
\hline \multirow[b]{2}{*}{ Domain } & \multicolumn{3}{|c|}{$\begin{array}{l}\text { Univariate meta-regression analysis } \\
\text { based on trials reporting primary } \\
\text { endpoint }\end{array}$} & \multicolumn{2}{|c|}{$\begin{array}{l}\text { Univariate meta-regression analysis based on trials } \\
\text { reporting all four domains significantly affecting } \\
\text { primary endpoint** }(k=15)\end{array}$} & \multicolumn{2}{|c|}{$\begin{array}{l}\text { Multivariable meta-regression analysis based } \\
\text { on trials reporting all four domains significantly } \\
\text { affecting primary endpoint** }(k=15)\end{array}$} \\
\hline & k & OR $(95 \% \mathrm{Cl})$ & $P$ value & OR $(95 \% \mathrm{Cl})$ & $P$ value & OR $(95 \% \mathrm{Cl})$ & $P$ value \\
\hline Overall* & 39 & $3.26(2.58,4.13)$ & $<0.001$ & $3.72(2.92,4.74)$ & $<0.001$ & & \\
\hline PF & 31 & $2.79(1.58,4.90)$ & 0.001 & $3.9(1.67,9.15)$ & 0.005 & $0.56(0.27,1.16)$ & 0.105 \\
\hline Pain & 27 & $2.11(1.45,3.06)$ & $<0.001$ & $5.6(2.4,13.15)$ & 0.001 & $5.19(2.28,11.77)$ & 0.001 \\
\hline SM & 31 & $1.20(0.94,1.5)$ & 0.142 & $2.25(1.27,3.99)$ & 0.009 & $1.03(0.64,1.68)$ & 0.883 \\
\hline SS & 22 & $1.29(0.81,2.06)$ & 0.268 & $1.88(0.62,1.56)$ & 0.227 & - & - \\
\hline PGA & 20 & $2.15(1.41,3.30)$ & 0.001 & $2.58(1.53,4.34)$ & 0.002 & $1.87(1.14,3.07)$ & 0.018 \\
\hline $\mathrm{PJ} / \mathrm{E}$ & 10 & $8.14(0.36,186.27)$ & 0.161 & $16.99(0.00,3687$ & 0.403 & - & - \\
\hline APR & 23 & $1.68(1.05,2.68)$ & 0.031 & $1.61(0.99,2.63)$ & 0.054 & $0.86(0.58,1.29)$ & 0.381 \\
\hline$S R$ & - & - & - & - & - & - & - \\
\hline Fatigue & 4 & $2.59(0.00,1360)$ & 0.581 & - & - & - & - \\
\hline
\end{tabular}

*Based on trials reporting primary outcome; PF physical function, SM spinal mobility, SS spinal stiffness, APR acute phase reactants, SR spine radiographs **PF, Pain, PGA, APR 
the OR for achieving primary outcome, regardless of analysis.

\section{Discussion}

This meta-research study aimed to assess the effect of interventions for axSpA according to each core domain in the existing ASAS/OMERACT-endorsed core outcome set. The eligible studies reported data only for patients with $r$-axSpA. The most frequent domains assessed in the included trials were SM and pain, which are considered prominent features for axSpA [76]. Overall outcome reporting was surprisingly good for SMARD/physical therapy trials, especially considering that most of the included studies were published prior to implementation of the COS.

The overall reporting for the included DC-ART trials was sparse. Surprisingly, none of the trials measured all the nine proposed domains. Fifteen $(30 \%)$ of the included studies were published before the COS was suggested by ASAS and endorsed by OMERACT, possibly explaining the lack of measured domains.

We found that all interventions, both nonpharmacological and pharmacological, when compared to control, resulted in an overall statistically significant reduction in pain related to axSpA, SS, fatigue, and APRs and an improvement in PF, SM, and PGA in axSpA trials. Due to our broad eligibility criteria where the type of interventions varied greatly among RCTs, the high between-study heterogeneity observed was not unexpected. However, type of intervention did not result in significant change in $\tau^{2}$ for all the domains. For the domain "PF," the overall effect size was moderate regardless of type of intervention. Our meta-analyses provided evidence that interventions in axSpA trials did not result in an overall reduction in swollen peripheral joint count/enthesitis index (PJ/E) or spinal progression more than placebo. However, when stratifying on type of interventions, it seemed that biological treatment had a larger effect on reducing the number of swollen PJ/E. However, one should be cautious to conclude that biologics are superior to other pharmacologicals for treating inflammation in $\mathrm{PJ} / \mathrm{E}$, as our meta-analysis included only a limited number of trials. Radiographic progression was measured in only two trials and fully reported in one. Given that most trials spanned 26 weeks or less, it is not surprising that they did not measure radiographic progression. MRI is an important imaging tool to assess axSpA, especially early in the disease course, before radiographic damage is apparent. Adding magnetic resonance imaging (MRI) to the domain "spine radiographs" could prove useful, as MRI is commonly used in short-term axSpA trials [77]. However, there is currently no consensus on how to monitor treatment response using MRI modalities in axSpA patients [78].

For transparency, we believe all domains and instruments used in trials should be reported. We found that domains and instruments sometimes were used but not reported separately. For example, the domain "fatigue," which is included in the Bath Ankylosing Spondylitis Disease Activity Index (BASDAI), was rarely reported separately, whereas the domain "spinal stiffness," also included in BASDAI, was reported in half of all the studies.

As with previous findings, this meta-epidemiological study found that pain and PGA are important predictors for treatment responses in axSpA trials [79], thus emphasizing the value of reporting core domains separately.

Outcome reporting bias (ORB) can affect the quality of evidence within a systematic review and meta-analysis [80]. We found a high suspicion of selective ORB in eight $(16 \%)$ of the individual included RCTs. In most cases, it was not possible to make a clear judgment on reporting bias due to the lack of published protocols in this context. Where protocols were available, there was no evidence of selective reporting. If a composite outcome (e.g., BASDAI) was reported but no data on any of the individual core outcome measurements (e.g., fatigue) were reported, then we judged ORB as low risk; it might not have been the trialists' intention to analyze the individual core outcomes separately. If a study reported some of the outcomes from the composite outcome measurement, then we judged ORB as a high risk, as it is likely that all the core outcome measurements were analyzed but some were not reported because of nonsignificant results. In many of the individual trials, all of the outcome domains were not mentioned, thereby requiring clinical judgment to decide whether the outcome of interest was likely to have been measured for a particular trial.

A limitation of this study was that we did not contact the trialists to determine whether outcomes were measured; many of the studies were published over 15 years ago, and it would have been difficult to locate the trialists. Another limitation of this study is that our results are based on axSpA trials included in Cochrane reviews, and therefore, we did not have control over the literature searches used. However, Cochrane reviews are known for the quality of their searches, and we consider the trials included in our study to be representative and our results to be generalizable. We used the SMDs to metaanalyze outcomes involving the same or similar constructs. We did not include absolute changes and reported in units/percentages of the most common instruments that the clinicians will understand. However, SMD is more generalizing and can be interpreted using a general rule of thumb reported by Cohen, in 
which an SMD of 0.2 represents a small effect, an SMD of 0.5 represents a medium effect, and an SMD of 0.8 or larger represents a large effect [12].

\section{Conclusions}

Although all types of axSpA conditions were eligible, the analyses were limited to patients with $r$-axial SpA (AS), since none of the eligible studies included patients with non-radiographic axSpA which could be either be perceived as a limitation or simply a consequence of the axSpA history reflected in the existing Cochrane reviews. Consistent outcome reporting for DC-ART trials was poor. The most responsible core domains for achieving success in meeting the primary objective per trial were pain and PGA. Our findings support that PGA and pain give us a more holistic assessment of disease beyond objective measures of spinal inflammation.

Outcome reporting bias and "missing data" could be reduced by implementing the endorsed ASAS/OMERACT COS of outcomes-and thereby improving the precision of results in meta-analyses.

\section{Supplementary information}

Supplementary information accompanies this paper at https://doi.org/10. 1186/s13075-020-02262-4.

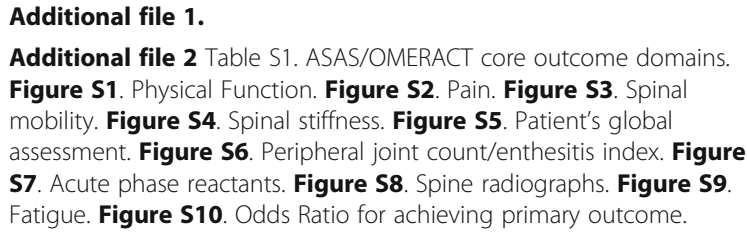

\begin{abstract}
Abbreviations
APR: Acute phase reactant; ASAS: Assessment of SpondyloArthritis international Society; axSpA: Axial spondyloarthritis; BASDAl: Bath Ankylosing Spondylitis Disease Activity Index; Cl: Confidence interval; COS: Core outcome set; CRP: C-reactive protein; ES: Effect size; MTX: Methotrexate; NP: Non-pharmacological; nr: Non-radiographic; NSAIDs: Non-steroidal antiinflammatory drugs; OMERACT: Outcome Measures in Rheumatology; OR: Odds ratio; ORB: Outcome reporting bias; ORBIT: Outcome reporting bias in trials; P: Pharmacological; PF: Physical function; PGA: Patient's global assessment; PJ/E: Peripheral joints and enthesitis index; PRISMA: Preferred Reporting Items for Systematic Reviews and Meta-Analyses; PRP: Patient research partner; r-: Radiographic; RCT: Randomized controlled trial; RoB: Risk of bias; SMD: Standardized mean difference; SpA: Spondyloarthritis; SM: Spinal mobility; SS: Spinal stiffness; SSZ: Sulfasalazine
\end{abstract}

\section{Acknowledgements}

The authors wish to thank patient research partner Maarten de Wit for his help in this study. The authors thank David Engel for proofreading of the final draft.

\section{Trial registration}

In accordance with current methodology, the protocol is available and registered on PROSPERO (registration number CRD42018091257) on March $29,2018$.

\section{Authors' contributions}

RAA have been involved in the literature search, data extraction, statistical analysis, and interpretation of data and drafted the manuscript. RC contributed substantially to the study conceptions and design, participated in the analysis interpretation, and critically revised the manuscript. LEK, XB, VS, PM, MdW, TE, IMJH, JK, GW, PT, LM, MB, and KE have been involved in the drafting and revision of the manuscript, and the authors approved the final manuscript for publication.

\section{Funding}

This study was supported by (1) the Danish Rheumatism Association, who provided financial support to running costs (grant number R144-A4136); (2) University of Southern Denmark, who provided a Faculty Scholarship; (3) Region Southern Denmark (grant number 15/50960) who provided a scholarship; and (4) the Department of Medicine, Odense University Hospital, Svendborg, who provided financial support to scientific personnel. (5) Carl Hansen \& Søn are acknowledged for financial support for purchasing computer and software; (6) Erna Hamiltons fond and (7) Else Poulsens fond (grant number 40-A1890) are acknowledged for grants, enabling the collection of the biological sample material; (8) the Parker Institute, Bispebjerg and Frederiksberg Hospital, is supported by a core grant from the Oak Foundation (OCAY-13-309). The sponsors were not involved in the study design, analyses or interpretation of results, and had no influence on the publishing of data.

\section{Availability of data and materials}

The data that support the findings of this study are available from the corresponding author $(\mathrm{RC})$ upon reasonable request.

Ethics approval and consent to participate

This is a meta-research study; ethical review is not required.

\section{Consent for publication}

Not applicable.

\section{Competing interests}

The authors declare that they have no competing interests.

\section{Author details}

${ }^{1}$ Department of Medicine, Section of Rheumatology, Odense University Hospital, Svendborg and University of Southern Denmark, Odense, Denmark.

${ }^{2}$ Musculoskeletal Statistics Unit, the Parker Institute, Bispebjerg and Frederiksberg Hospital, University Hospital, Copenhagen F, Denmark. ${ }^{3}$ Rheumazentrum Ruhrgebiet Herne, Ruhr-University Bochum, Bochum, Germany. ${ }^{4}$ Division Immunology/Rheumatology, Stanford University, Palo Alto, CA, USA. ${ }^{5}$ Swedish Medical Centre/Providence St. Joseph Health and University of Washington, Seattle, USA. ${ }^{6}$ Amsterdam, the Netherlands. ${ }^{7}$ Research Unit of Rheumatology, Department of Clinical Research, University of Southern Denmark, Odense University Hospital, Odense, Denmark. ${ }^{8}$ Centre for Biostatistics, Manchester Academic Health Science, Manchester, UK.

${ }^{9}$ Department of Medicine, University of Ottawa, Ottawa, Ontario, Canada.

${ }^{10}$ Faculty of Medicine, University of Ottawa, Ottawa, Ontario, Canada.

${ }^{11}$ Department of Epidemiology \& Biostatistics, Amsterdam Rheumatology and Immunology Center, Amsterdam University Medical Centers, Vrije Universiteit, Amsterdam, the Netherlands. ${ }^{12}$ Cardiovascular Research Unit, Odense University Hospital, Svendborg, Denmark.

Received: 30 March 2020 Accepted: 6 July 2020

Published online: 25 July 2020

\section{References}

1. Boers M, Kirwan JR, Wells G, Beaton D, Gossec L, d'Agostino MA, et al. Developing core outcome measurement sets for clinical trials: OMERACT filter 2.0. J Clin Epidemiol. 2014;67(7):745-53

2. Prinsen CA, Vohra S, Rose MR, Boers M, Tugwell P, Clarke M, et al. How to select outcome measurement instruments for outcomes included in a "Core Outcome Set" - a practical guideline. Trials. 2016;17(1):449.

3. Sieper J, Rudwaleit M, Baraliakos X, Brandt J, Braun J, Burgos-Vargas R, et al. The Assessment of SpondyloArthritis international Society (ASAS) handbook: a guide to assess spondyloarthritis. Ann Rheum Dis. 2009;68(Suppl 2):ii1-44.

4. van der Heijde D, Calin A, Dougados M, Khan MA, van der Linden S, Bellamy N. Selection of instruments in the core set for DC-ART, SMARD, physical therapy, and clinical record keeping in ankylosing spondylitis. Progress report of the ASAS Working Group. Assessments in Ankylosing Spondylitis. J Rheumatol. 1999;26(4):951-4. 
5. van der Heijde D, Bellamy N, Calin A, Dougados M, Khan MA, van der Linden S. Preliminary core sets for endpoints in ankylosing spondylitis Assessments in Ankylosing Spondylitis Working Group. J Rheumatol. 1997; 24(11):2225-9.

6. Shamseer L, Moher D, Clarke M, Ghersi D, Liberati A, Petticrew M, et al. Preferred reporting items for systematic review and meta-analysis protocols (PRISMA-P) 2015: elaboration and explanation. BMJ. 2015;350:g7647.

7. Liberati A, Altman DG, Tetzlaff J, Mulrow C, Gotzsche PC, loannidis JP, et al. The PRISMA statement for reporting systematic reviews and meta-analyses of studies that evaluate healthcare interventions: explanation and elaboration. BMJ. 2009;339:b2700.

8. Higgins JPT, Green S, eds. Cochrane handbook for systematic reviews of interventions. Wiley; 2008.

9. Higgins JP, Altman DG, Gotzsche PC, Juni P, Moher D, Oxman AD, et al. The Cochrane Collaboration's tool for assessing risk of bias in randomised trials. BMJ. 2011;343:d5928.

10. Tarp S, Bartels EM, Bliddal H, Furst DE, Boers M, Danneskiold-Samsoe B, et al. Effect of nonsteroidal antiinflammatory drugs on the C-reactive protein level in rheumatoid arthritis: a meta-analysis of randomized controlled trials. Arthritis Rheum. 2012;64(11):3511-21.

11. Normand SL. Meta-analysis: formulating, evaluating, combining, and reporting. Stat Med. 1999;18(3):321-59.

12. Bliddal $H$, Christensen $R$. The treatment and prevention of knee osteoarthritis: a tool for clinical decision-making. Expert Opin Pharmacother. 2009;10(11):1793-804

13. Higgins JP, Thompson SG, Deeks JJ, Altman DG. Measuring inconsistency in meta-analyses. BMJ. 2003;327(7414):557-60.

14. de Wit MP, Berlo SE, Aanerud GJ, Aletaha D, Bijlsma JW, Croucher L, et al. European League Against Rheumatism recommendations for the inclusion of patient representatives in scientific projects. Ann Rheum Dis. 2011;70(5): 722-6.

15. Moore RA, Derry S, Aldington D, Wiffen PJ. Single dose oral analgesics for acute postoperative pain in adults - an overview of Cochrane reviews. Cochrane Database Syst Rev. 2015(9). https://doi.org/10.1002/14651858. CD008659.pub3.

16. Kroon FP, van der Burg LR, Ramiro S, Landewe RB, Buchbinder R, Falzon L, et al. Non-steroidal anti-inflammatory drugs (NSAIDs) for axial spondyloarthritis (ankylosing spondylitis and non-radiographic axial spondyloarthritis). Cochrane Database Syst Rev. 2015(7). https://doi.org/10. 1002/14651858.CD010952.pub2.

17. Maxwell $\sqcup$, Zochling J, Boonen A, Singh JA, Veras MM, Tanjong Ghogomu E, et al. TNF-alpha inhibitors for ankylosing spondylitis. Cochrane Database Syst Rev. 2015(4). https://doi.org/10.1002/14651858.CD005468.pub2.

18. Chen J, Lin S, Liu C. Sulfasalazine for ankylosing spondylitis. Cochrane Database Syst Rev. 2014(11). https:/doi.org/10.1002/14651858.CD004800.pub3.

19. Chen J, Veras MM, Liu C, Lin J. Methotrexate for ankylosing spondylitis. Cochrane Database Syst Rev. 2013(2). https://doi.org/10.1002/14651858. CD004524.pub4

20. Dagfinrud H, Kvien TK, Hagen KB. Physiotherapy interventions for ankylosing spondylitis. Cochrane Database Syst Rev. 2008(1). https://doi.org/10.1002/ 14651858.CD002822.pub3.

21. Radner H, Ramiro S, Buchbinder R, Landewe RB, van der Heijde D, Aletaha D. Pain management for inflammatory arthritis (rheumatoid arthritis, psoriatic arthritis, ankylosing spondylitis and other spondylarthritis) and gastrointestinal or liver comorbidity. Cochrane Database Syst Rev. 2012;1: Cd008951.

22. Ramiro S, Radner $H$, van der Heijde $D$, van Tubergen A, Buchbinder $\mathrm{R}$, Aletaha $\mathrm{D}$, et al. Combination therapy for pain management in inflammatory arthritis (rheumatoid arthritis, ankylosing spondylitis, psoriatic arthritis, other spondyloarthritis). Cochrane Database Syst Rev. 2011(10).DOI: https://doi.org/10.1002/14651858.CD008886.pub2.

23. Colebatch AN, Marks JL, Edwards CJ. Safety of non-steroidal antiinflammatory drugs, including aspirin and paracetamol (acetaminophen) in people receiving methotrexate for inflammatory arthritis (rheumatoid arthritis, ankylosing spondylitis, psoriatic arthritis, other spondyloarthritis). Cochrane Database Syst Rev. 2011(11). https://doi.org/10.1002/14651858.CD008872.pub2.

24. Hoving JL, Lacaille D, Urquhart DM, Hannu TJ, Sluiter JK, Frings-Dresen MH. Non-pharmacological interventions for preventing job loss in workers with inflammatory arthritis. The Cochrane database of systematic reviews. 2014(11). https://doi.org/10.1002/14651858.CD010208.pub2.
25. Regnaux JP, Davergne T, Palazzo C, Roren A, Rannou F, Boutron I, et al. Exercise programmes for ankylosing spondylitis. Cochrane Database Syst Rev. 2019;10:Cd011321.

26. Spencer S, Martindale JH, MacPhie E, Montgomery P. Self management programme for ankylosing spondylitis. Cochrane Database Syst Rev. 2015. Issue 12. Art. No.: CD006977. https://doi.org/10.1002/14651858.CD006977.pub2.

27. Barkhuizen A, Steinfeld S, Robbins J, West C, Coombs J, Zwillich S. Celecoxib is efficacious and well tolerated in treating signs and symptoms of ankylosing spondylitis. J Rheumatol. 2006;33(9):1805-12.

28. Feltelius N, Hallgren R. Sulphasalazine in ankylosing spondylitis. Ann Rheum Dis. 1986:45(5):396-9.

29. Kirwan J, Edwards A, Huitfeldt B, Thompson P, Currey H. The course of established ankylosing spondylitis and the effects of sulphasalazine over 3 years. Br J Rheumatol. 1993;32(8):729-33.

30. Lim HJ, Moon Yl, Lee MS. Effects of home-based daily exercise therapy on joint mobility, daily activity, pain, and depression in patients with ankylosing spondylitis. Rheumatol Int. 2005;25(3):225-9.

31. Helliwell PS, Abbott CA, Chamberlain MA. A randomised trial of three different physiotherapy regimes in ankylosing spondylitis. Physiotherapy. 1996;82(2):85-90.

32. van der Heijde D, Da Silva JC, Dougados $M$, Geher $P$, van der HorstBruinsma I, Juanola X, et al. Etanercept $50 \mathrm{mg}$ once weekly is as effective as 25 mg twice weekly in patients with ankylosing spondylitis. Ann Rheum Dis. 2006;65(12):1572-7.

33. Huang F, Zhang J, Huang JL, Wu DH, Li ZG, Chen SL, et al. A multicenter, double-blind, placebo-controlled, randomized, phase III clinical study of etanercept in treatment of ankylosing spondylitis. Zhonghua nei ke za zhi. 2010;49(9):741-5.

34. Taylor HG, Beswick EJ, Dawes PT. Sulphasalazine in ankylosing spondylitis. A radiological, clinical and laboratory assessment. Clin Rheumatol. 1991;10(1): 43-8.

35. Altan L, Bingol U, Karakoc Y, Aydiner S, Yurtkuran M, Yurtkuran M. Clinical investigation of methotrexate in the treatment of ankylosing spondylitis. Scand J Rheumatol. 2001:30(5):255-9.

36. Braun J, Brandt J, Listing J, Zink A, Alten R, Golder W, et al. Treatment of active ankylosing spondylitis with infliximab: a randomised controlled multicentre trial. Lancet. 2002;359(9313):1187-93.

37. Brandt J, Khariouzov A, Listing J, Haibel H, Sorensen $H$, Grassnickel L, et al. Six-month results of a double-blind, placebo-controlled trial of etanercept treatment in patients with active ankylosing spondylitis. Arthritis Rheum. 2003:48(6):1667-75

38. Gonzalez-Lopez L, Garcia-Gonzalez A, Vazquez-Del-Mercado M, Munoz-Valle JF, Gamez-Nava Jl. Efficacy of methotrexate in ankylosing spondylitis: a randomized, double blind, placebo controlled trial. J Rheumatol. 2004;31(8): 1568-74.

39. Dougados M, Braun J, Szanto S, Combe B, Elbaz M, Geher P, et al. Efficacy of etanercept on rheumatic signs and pulmonary function tests in advanced ankylosing spondylitis: results of a randomised double-blind placebocontrolled study (SPINE). Ann Rheum Dis. 2011:70(5):799-804.

40. Hu Z, Xu M, Li Q, Lin Z, Liao Z, Cao S, et al. Adalimumab significantly reduces inflammation and serum DKK-1 level but increases fatty deposition in lumbar spine in active ankylosing spondylitis. Int J Rheum Dis. 2012;15(4): 358-65.

41. Dougados M, Nguyen M, Caporal R, Legeais J, Bouxin-Sauzet A, PellegriGuegnault B, et al. Ximoprofen in ankylosing spondylitis. A double blind placebo controlled dose ranging study. Scand J Rheumatol. 1994;23(5):243-8.

42. Dougados M, Gueguen A, Nakache JP, Velicitat $P$, Veys EM, Zeidler $H$, et al. Ankylosing spondylitis: what is the optimum duration of a clinical study? A one year versus a 6 weeks non-steroidal anti-inflammatory drug trial. Rheumatology. 1999;38(3):235-44.

43. Dougados M, Behier JM, Jolchine I, Calin A, van der Heijde D, Olivieri I, et al. Efficacy of celecoxib, a cyclooxygenase 2-specific inhibitor, in the treatment of ankylosing spondylitis: a six-week controlled study with comparison against placebo and against a conventional nonsteroidal antiinflammatory drug. Arthritis Rheum. 2001;44(1):180-5.

44. van der Heijde D, Dijkmans B, Geusens P, Sieper J, DeWoody K, Williamson $P$, et al. Efficacy and safety of infliximab in patients with ankylosing spondylitis: results of a randomized, placebo-controlled trial (ASSERT). Arthritis Rheum. 2005:52(2):582-91.

45. Kraag G, Stokes B, Groh J, Helewa A, Goldsmith C. The effects of comprehensive home physiotherapy and supervision on patients with 
ankylosing spondylitis--a randomized controlled trial. J Rheumatol. 1990; 17(2):228-33.

46. Hidding A, van der Linden S, Boers M, Gielen X, de Witte L, Kester A, et al. Is group physical therapy superior to individualized therapy in ankylosing spondylitis? A randomized controlled trial. Arthritis Care Res. 1993;6(3):117-25.

47. van Tubergen $A$, Landewe $R$, van der Heijde $D$, Hidding A, Wolter $N$, Asscher M, et al. Combined spa-exercise therapy is effective in patients with ankylosing spondylitis: a randomized controlled trial. Arthritis Rheum. 2001; 45(5):430-8.

48. Sweeney S, Taylor G, Calin A. The effect of a home based exercise intervention package on outcome in ankylosing spondylitis: a randomized controlled trial. J Rheumatol. 2002;29(4):763-6.

49. Analay Y, Ozcan E, Karan A, Diracoglu D, Aydin R. The effectiveness of intensive group exercise on patients with ankylosing spondylitis. Clin Rehabil. 2003;17(6):631-6.

50. Codish S, Dobrovinsky S, Abu Shakra M, Flusser D, Sukenik S. Spa therapy for ankylosing spondylltis at the Dead Sea. IMAJ. 2005;7(7):443-6.

51. Fernandez-de-Las-Penas C, Alonso-Blanco C, Morales-Cabezas M, Miangolarra-Page JC. Two exercise interventions for the management of patients with ankylosing spondylitis: a randomized controlled trial. Am J Phys Med Rehabil. 2005;84(6):407-19.

52. Ince G, Sarpel T, Durgun B, Erdogan S. Effects of a multimodal exercise program for people with ankylosing spondylitis. Phys Ther. 2006;86(7):924-35.

53. Altan L, Bingol U, Aslan M, Yurtkuran M. The effect of balneotherapy on patients with ankylosing spondylitis. Scand J Rheumatol. 2006;35(4):283-9.

54. Dougados M, Boumier P, Amor B. Sulphasalazine in ankylosing spondylitis: a double blind controlled study in 60 patients. Br Med J (Clin Res Ed). 1986; 293(6552):911-4.

55. Nissila M, Lehtinen K, Leirisalo-Repo M, Luukkainen R, Mutru O, Yli-Kerttula $U$. Sulfasalazine in the treatment of ankylosing spondylitis. A twenty-sixweek, placebo-controlled clinical trial. Arthritis Rheum. 1988;31(9):1111-6.

56. Davis MJ, Dawes PT, Beswick E, Lewin IV, Stanworth DR. Sulphasalazine therapy in ankylosing spondylitis: its effect on disease activity, immunoglobulin $\mathrm{A}$ and the complex immunoglobulin A-alpha-1-antitrypsin. Br J Rheumatol. 1989;28(5):410-3.

57. Winkler V. [Sulfasalazine therapy in spondylarthritis ankylopoietica]. Orv Hetil. 1989;130(2):77-81.

58. Corkill MM, Jobanputra P, Gibson T, Macfarlane DG. A controlled trial of sulphasalazine treatment of chronic ankylosing spondylitis: failure to demonstrate a clinical effect. Br J Rheumatol. 1990;29(1):41-5.

59. Krajnc I. [Sulfasalazine in the treatment of ankylosing spondylitis]. Lijecnicki vjesnik. 1990;112(5-6):171-4.

60. Clegg DO, Reda DJ, Weisman MH, Blackburn WD, Cush JJ, Cannon GW, et al. Comparison of sulfasalazine and placebo in the treatment of ankylosing spondylitis. A Department of Veterans Affairs Cooperative Study. Arthritis Rheum. 1996;39(12):2004-12.

61. Gorman JD, Sack KE, Davis JC, Jr. Treatment of ankylosing spondylitis by inhibition of tumor necrosis factor alpha. N Engl J Med. 2002;346(18):1349-56.

62. Roychowdhury B, Bintley-Bagot S, Bulgen DY, Thompson RN, Tunn EJ, Moots RJ. Is methotrexate effective in ankylosing spondylitis? Rheumatology (Oxford). 2002;41(11):1330-2.

63. Schmidt WA, Wierth S, Milleck D, Droste U, Gromnica-lhle E. [Sulfasalazine in ankylosing spondylitis: a prospective, randomized, double-blind placebocontrolled study and comparison with other controlled studies]. Z Rheumatol. 2002;61(2):159-67.

64. Davis JC, Jr., Van Der Heijde D, Braun J, Dougados M, Cush J, Clegg DO, et al. Recombinant human tumor necrosis factor receptor (etanercept) for treating ankylosing spondylitis: a randomized, controlled trial. Arthritis Rheum. 2003:48(11):3230-6.

65. Calin A, Dijkmans BAC, Emery P, Hakala M, Kalden J, Leirisalo-Repo M, et al. Outcomes of a multicentre randomised clinical trial of etanercept to treat ankylosing spondylitis. Ann Rheum Dis. 2004;63(12):1594-600.

66. Marzo-Ortega H, McGonagle D, Jarrett S, Haugeberg G, Hensor E, O'Connor P, et al. Infliximab in combination with methotrexate in active ankylosing spondylitis: a clinical and imaging study. Ann Rheum Dis. 2005;64(11):1568-75.

67. van der Heijde D, Kivitz A, Schiff MH, Sieper J, Dijkmans BA, Braun J, et al. Efficacy and safety of adalimumab in patients with ankylosing spondylitis: results of a multicenter, randomized, double-blind, placebo-controlled trial. Arthritis Rheum. 2006;54(7):2136-46.

68. Lambert RG, Salonen D, Rahman P, Inman RD, Wong RL, Einstein SG, et al. Adalimumab significantly reduces both spinal and sacroiliac joint inflammation in patients with ankylosing spondylitis: a multicenter, randomized, double-blind, placebo-controlled study. Arthritis Rheum. 2007; 56(12):4005-14.

69. Inman RD, Davis JC, Jr., Heijde D, Diekman L, Sieper J, Kim Sl, et al. Efficacy and safety of golimumab in patients with ankylosing spondylitis: results of a randomized, double-blind, placebo-controlled, phase III trial. Arthritis Rheum. 2008:58(11):3402-12.

70. Barkham N, Coates LC, Keen H, Hensor E, Fraser A, Redmond A, et al. Double-blind placebo-controlled trial of etanercept in the prevention of work disability in ankylosing spondylitis.Ann Rheum Dis. 2010;69(11):1926-8.

71. Inman RD, Maksymowych WP. A Double-blind, Placebo-controlled Trial of Low Dose Infliximab in Ankylosing Spondylitis. J Rheumatol. 2010;37(6): 1203-10.

72. Braun J, van der Horst-Bruinsma IE, Huang F, Burgos-Vargas R, Vlahos B, Koenig AS, et al. Clinical efficacy and safety of etanercept versus sulfasalazine in patients with ankylosing spondylitis: a randomized, doubleblind trial. Arthritis Rheum. 2011;63(6):1543-51.

73. Navarro-Sarabia F, Fernandez-Sueiro JL, Torre-Alonso JC, Gratacos J, Queiro R, Gonzalez C, et al. High-dose etanercept in ankylosing spondylitis: results of a 12-week randomized, double blind, controlled multicentre study (LOADET study). Rheumatology (Oxford). 2011;50(10):1828-37.

74. Bao C, Huang F, Khan MA, Fei K, Wu Z, Han C, et al. Safety and efficacy of golimumab in Chinese patients with active ankylosing spondylitis: 1-year results of a multicentre, randomized, double-blind, placebo-controlled phase III trial. Rheumatology (Oxford). 2014;53(9):1654-63.

75. Huang $F, G u J, Z h u, P, B a o ~ C, X u J, X u ~ H$, et al. Efficacy and safety of adalimumab in Chinese adults with active ankylosing spondylitis: results of a randomised, controlled trial. Ann Rheum Dis. 2014;73(3):587-94.

76. Sieper J, Braun J, Dougados M, Baeten D. Axial spondyloarthritis. Nat Revi Dis Prim. 2015:1:15013.

77. Molto A, Gossec L, Lefevre-Colau MM, Foltz V, Beaufort R, Laredo JD, et al. Evaluation of the performances of 'typical' imaging abnormalities of axial spondyloarthritis: results of the cross-sectional ILOS-DESIR study. RMD Open. 2019;5(1):e000918

78. Schwartzman M, Maksymowych WP. Is there a role for MRI to establish treatment indications and effectively monitor response in patients with axial spondyloarthritis? Rheum Dis Clin N Am. 2019:45(3):341-58.

79. Mease P, Walsh JA, Baraliakos X, Inman R, de Vlam K, Wei JC, et al. Translating improvements with ixekizumab in clinical trial outcomes into clinical practice: ASAS40, pain, fatigue, and sleep in ankylosing spondylitis. Rheum Ther. 2019;6(3):435-50. https://doi.org/10.1007/s40744-019-0165-3.

80. Kirkham JJ, Gargon E, Clarke M, Williamson PR. Can a core outcome set improve the quality of systematic reviews?--a survey of the Co-ordinating Editors of Cochrane Review Groups. Trials. 2013;14:21.

\section{Publisher's Note}

Springer Nature remains neutral with regard to jurisdictional claims in published maps and institutional affiliations. 